\title{
Comparison of Aquatic Treadmill and Anti-Gravity Treadmill Gait Training to Improve Balance and Gait Abilities in Stroke Patients
}

\author{
Jae Ho Park', Yi Jung Chung ${ }^{2}$ \\ 'Department of Physical Therapy, The Graduate School, Sahmyook University, Seoul; ${ }^{2}$ Department of Physical Therapy, College of Health Science and \\ Social Welfare, Sahmyook University, Seoul, Korea
}

Purpose: The purpose of this study was to compare to aquatic treadmill and anti-gravity treadmill gait training to improve balance and gait abilities in stroke patients.

Methods: All subjects were randomly divided into three groups where nine subjects were in the aquatic treadmill group, eight subjects in the anti-gravity treadmill group, and ten subjects in the control group. Subjects in the aquatic treadmill group and the anti-gravity treadmill group received gait training during 30 minutes, with 3 sessions per week for 4 weeks, and subjects in all groups received conventional physical therapy during 30 minutes, with 5 sessions per week for 4 weeks. All subjects were assessed with the Berg balance scale (BBS), timed up and go test (TUG) and 10-meter walk test (10MWT) pre and post intervention.

Results: Results showed that BBS, TUG and 10MWT scores significantly improved post-intervention $(p<0.05)$, and the control group also had significantly improved in all areas pre-post intervention $(p<0.05)$. In addition, it has been confirmed that aquatic treadmill group and anti-gravity treadmill group had significantly improved in BBS, TUG and 10MWT scores compared with the control group $(p<0.05)$. However, no significant difference was found in the comparison between the aquatic treadmill and the anti-gravity treadmill group. Conclusion: Finding of this study suggested that aquatic treadmill and anti-gravity treadmill improves balance and gait abilities in stroke patients.

Keywords: Balance, Gait, Rehabilitation, Stroke

\section{INTRODUCTION}

Stroke patients suffer from asymmetric weight support and body imbalance, resulting in a decrease in weight transfer capacity and a problem of functional performance. In particular, it is known that the imbalance of strength of low extremity muscle has a great effect on posture control and walking ability. ${ }^{2}$ As a result, stroke patients show secondary impairments such as movement restriction and falls. ${ }^{3}$ This impairment of gait ability is the most important factor in determining the dysfunction of stroke patients. ${ }^{4}$ Therefore, restoring the balance and walking ability of stroke patients is essential for functional independence in everyday life. ${ }^{5}$

Balance refers to the ability to maintain posture stability by placing a center of gravity (COG) on the support surface. ${ }^{6}$ The gait abili- ty, which represents the patterned movement due to this balance ability, exhibits symmetrical movements in spatio-temporal. ${ }^{7}$ Park et al. ${ }^{8}$ refer to this method as an effective treatment method by providing the stability and exercise effects that cannot be obtained from the ground motion by using the special properties of water as a method of restoring this normal balance ability. It also noted that aquatic exercise provides muscle release and posture stability, which can lead to a rhythmical gait pattern compared to general gait training. ${ }^{9.10}$ Based on the results of Masumoto et al. ${ }^{11}$ we found that the application of underwater treadmill was the basis for a positive change in lower extremity muscle activity. In particular, Park et al. ${ }^{12}$ showed that underwater gait training has a very effective effect on gait symmetry compared with over-ground gait training. Arnold et al. ${ }^{13}$ and Hinman et al..$^{14}$ used the aquatic exercise program combined 
with underwater gait training to derive results that show the strength of lower extremity and balance ability. In addition, Jung et al. ${ }^{15}$ found that the stability of the lower extremity increases with the application of aquatic treadmill through three-dimensional motion analysis. Recently, the anti-gravity treadmill, which is mentioned as one of the methods of early gait training, is also said to be effective in improving walking ability through symmetrical gait. ${ }^{16}$ As a basis for this, Berthelsen et al. ${ }^{17}$ reported that early gait training using the anti-gravity treadmill was effective in posture stability and walking ability. Thus, there is an increased interest in underwater walking training and anti-gravity treadmill, which are very effective in this early gait training. Mercer et al. ${ }^{18}$ conducted a cross-sectional study by comparing the underwater walking training with the anti-gravity treadmill. Results showed no significant difference between the two interventions. It is considered that the short term period of experiment. And subjects were the general people. In other studies, most of the studies were applied to elite athletes or the general public, and studies on patients with nervous system injuries were rare. The purpose of this study is to compare the effect of aquatic treadmill and anti-gravity treadmill on balance ability and walking ability in stroke patients.

\section{METHODS}

\section{Subjects}

\section{1) Participants}

This study included a pretest-posttest control group design where the subjects were divided according into intervention methods, such as aquatic treadmill group, anti-gravity treadmill group, or control group. Twenty seven subjects who were admitted in ChungNam national university hospital in Daejeon. In addition, the subjects were not experienced in aquatic treadmill and anti-gravity treadmill. Stroke patients were composed of patients who had no overlapping diseases within the past 6 months after the onset of stroke to minimize the possibility of natural restoration. To minimize the selection bias, the following selection criteria were applied randomly to the three groups. The inclusion criteria were as follows: (1) diagnosis of stroke (after minimum 6 months), (2) ability to walk 10 minutes with or without an assistive device, (3) impairment of balance ability (maximum Berg balance scale score 45), (4) cognitive abilities enabling communication (minimum MMSE score 24), (5)

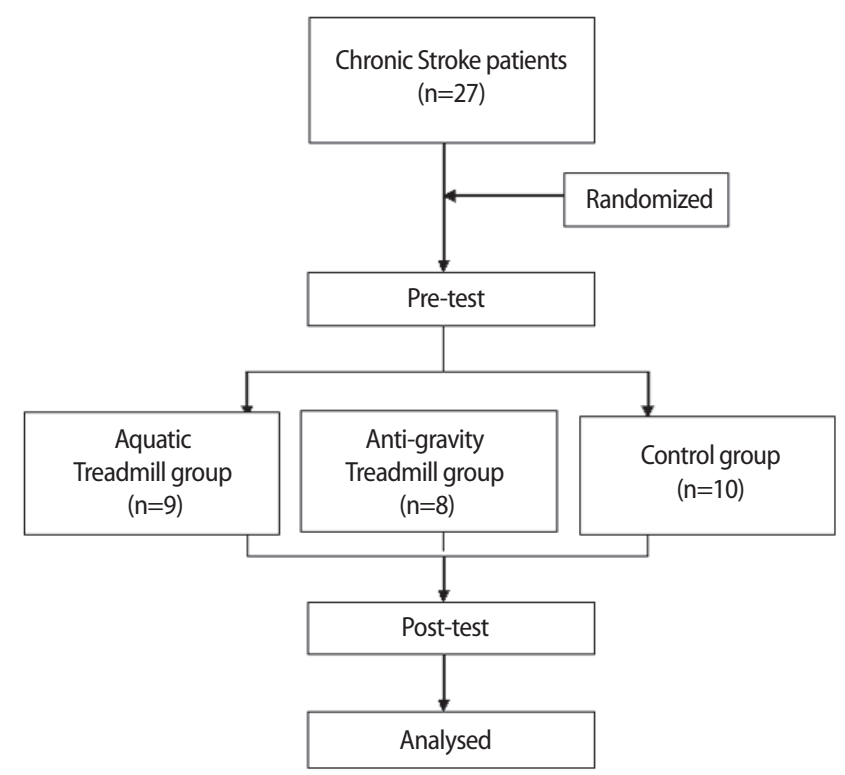

Figure 1. Experimental procedures.

medically stable and free of major cardiovascular or other medical conditions, and (6) no history of orthopedic surgery within the past 6 months and seizure. And the subjects who did not meet the above criteria were excluded. Exclusion criteria included those with visual or auditory deficits. The general characteristics of the participants are shown in Table 1. This study trained twenty seven patients for four weeks, but three patients were due to discharge. Three therapists intervened to conduct the study on a large number of patients. All groups measured balance abilities and gait abilities before intervention. For objective evaluation, four therapists with at least five years of career conducted the evaluation. This study was approved by the Institutional Review Board of Sahmyook University, and all participants signed informed consent forms after receiving a detailed explanation of the study (Figure 1).

\section{2) Intervention}

\section{(1) Aquatic treadmill group}

Underwater In this study, aquatic gait training was performed using aqua-track 2000. During aquatic gait training, it was conducted in a pool at chest height depth (xiphoid process) with water temperatures of 30 to $32^{\circ} \mathrm{C}$. For mediation of gait speed during underwater gait training, researchers used metronomes to adjust the tempo to the subject's comfortable walking speed. The intervention periods for the subjects were 4 weeks, 3 sessions a week, for 30 minutes each. Also, all patients who participated in the study were conducted con- 


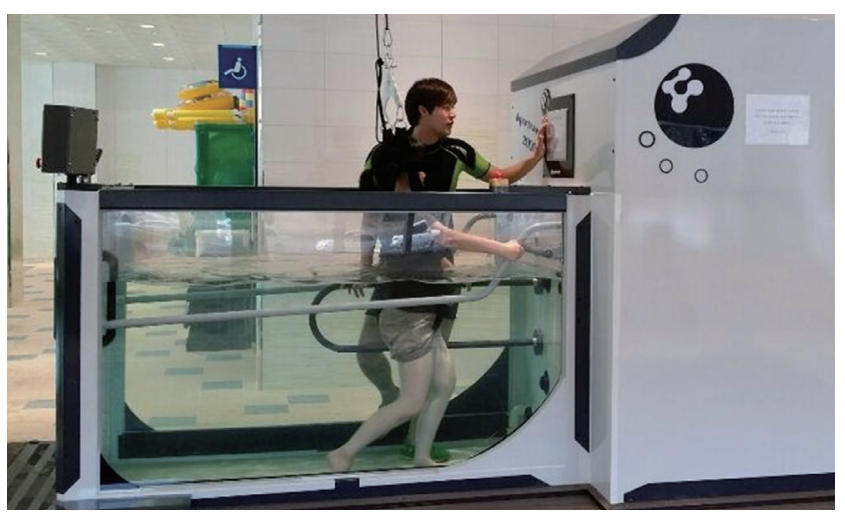

Figure 2. Aquatic treadmill gait training.

ventional physical therapy for 4 weeks, 5 sessions a week. For subject safety, guidance during training was provided by physical therapists with 3 more years of experience of aquatic exercise training and one assistant per subject as research assistants (Figure 2).

\section{(2) Anti-gravity treadmill group}

In this study, anti-gravity gait training was performed using Alter $\mathrm{G}$ Anti-Gravity Treadmill ${ }^{\oplus}$. We also applied to same method to the gait speed of the an-gravity treadmill as the aquatic treadmill (Figure 3).

\section{(3) Control group}

The control group performed general gait training using a treadmill. The training was conducted for 30 minutes 3 sessions a week for 4 weeks in same manner as the experimental group. All patients who participated in the study were conducted conventional physical therapy for 4 weeks, 5 sessions a week.

\section{Experimental methods}

\section{1) Measurement}

\section{(1) The Berg balance scale (BBS)}

The Berg balance scale is composed of 14 different items that can quantitatively evaluate the degree of balance and fall risk through direction observation. Items can be classified into three regions of sitting, standing, and postural changes, and each of the 14 items can be scored between $0-4$ points, with 56 points being the maximum score. A score of 45 or less indicates the need for the use of a cane or other gait assistive devices, a score of 41-44 indicates a low fall-risk, 21-40 indicates a higher fall risk, and 0-20 indicates a very high risk for falls and injuries. The evaluation assessed dynamic and static balance ability and takes approximately 15 minutes to complete. A

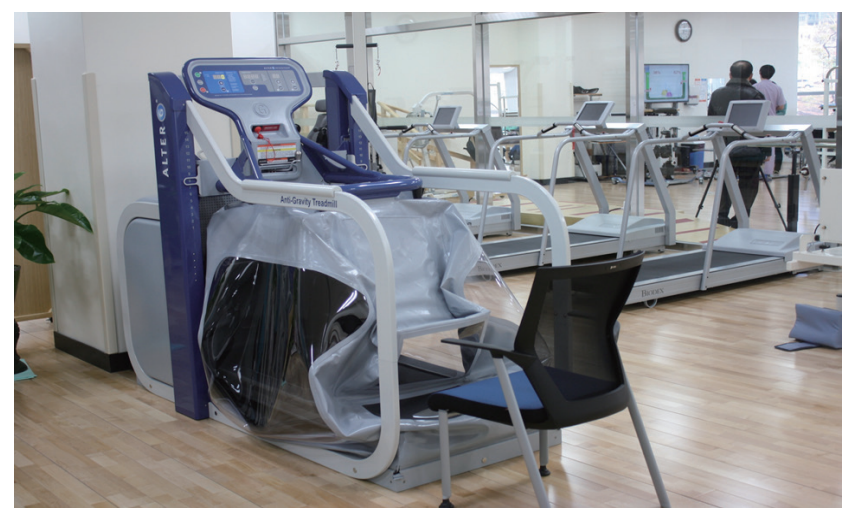

Figure 3. Anti-gravity treadmill gait training.

therapist with more than 3 years of clinical experience had performed the measurements prior to and after the intervention.

\section{(2) The timed up and go test (TUG)}

The TUG measures the time it takes for a subject to rise from a seated position at the "start" signal, walk up to the $3 \mathrm{~m}$ mark, and then return back to the chair until they are completely seated. It has a reliability of $r=0.99$ and a high inter-rater reliability of $r=0.98$. The mean value was obtained from a total of three measurements.

\section{(3) 10 meter walking test (10MWT)}

With the presence of gait disabilities due to neurological damage, the 10MWT is a standard test used to investigate the extent of gait ability.

\section{2) Data analysis}

This study used the PASW Statistics ver. 19.0 program (IBM Co., Armonk, NY, USA). A normality analysis was performed on the general characteristics of subjects and a paired t-test was performed to examine for changes pre and post-intervention for each group. A Kruskal-Wallis test was used to determine for statistically significant differences in balance and gait ability between groups after four weeks, a post-hoc analysis was performed with the Duncan method, and the significance level was set at $\mathrm{p}<0.05$.

\section{RESULTS}

\section{General characteristics and medical characteristics of subjects}

The general characteristics and medical characteristics of all subjects in the aquatic treadmill group, anti-gravity treadmill group 
Table 1. Characteristics of participants (clinical features)

\begin{tabular}{|c|c|c|c|c|c|}
\hline & Aquatic Treadmill $(n=9)$ & Anti-gravity Treadmill $(n=8)$ & Control $(n=10)$ & $\mathrm{F}$ & $\mathrm{p}$ \\
\hline Age (year) & $62.88 \pm 12.90$ & $66.37 \pm 10.28$ & $67.10 \pm 7.63$ & 0.18 & NS \\
\hline Height (cm) & $164.11 \pm 10.26$ & $159.12 \pm 7.33$ & $165.70 \pm 9.74$ & 0.32 & NS \\
\hline Weight (kg) & $63.77 \pm 9.14$ & $59.37 \pm 10.35$ & $62.80 \pm 9.94$ & 0.63 & NS \\
\hline Delay (month) & $7.22 \pm 1.09$ & $6.75 \pm 0.88$ & $7.60 \pm 0.84$ & 0.18 & NS \\
\hline MMSE-K & $27.33 \pm 1.41$ & $27.37 \pm 1.59$ & $27.40 \pm 0.98$ & 0.99 & NS \\
\hline \multicolumn{6}{|l|}{ Gender } \\
\hline Male & 6 & 4 & 5 & \multirow[t]{2}{*}{0.80} & \multirow[t]{2}{*}{ NS } \\
\hline Female & 3 & 4 & 5 & & \\
\hline \multicolumn{6}{|l|}{ Hemiplegic side } \\
\hline Left & 6 & 4 & 4 & \multirow[t]{2}{*}{0.80} & \multirow[t]{2}{*}{ NS } \\
\hline Right & 3 & 4 & 6 & & \\
\hline \multicolumn{6}{|l|}{ Mechanism } \\
\hline Haemorrhage & 1 & 1 & 4 & \multirow[t]{2}{*}{0.25} & \multirow[t]{2}{*}{ NS } \\
\hline Ischaemia & 8 & 7 & 6 & & \\
\hline
\end{tabular}

Aquatic treadmill: aquatic treadmill gait training group, Anti-gravity treadmill: anti-gravity treadmill gait training group.

Table 2. Changes in balance ability and gait ability of the participants in this study

\begin{tabular}{|c|c|c|c|c|c|c|c|c|}
\hline & \multicolumn{2}{|c|}{ Aquatic treadmill $(n=9)$} & \multicolumn{2}{|c|}{ Anti-gravity treadmill $(n=8)$} & \multicolumn{2}{|c|}{ Control $(n=10)$} & \multirow{2}{*}{$\mathrm{F}$} & \multirow{2}{*}{$p$} \\
\hline & Pre-test & Post-test & Pre-test & Post-test & Pre-test & Post-test & & \\
\hline BBS (score) & $40.22 \pm 2.48$ & $49.33 \pm 1.73$ & $40.88 \pm 2.80$ & $49.25 \pm 1.75$ & $40.80 \pm 1.68$ & $47.00 \pm 1.41$ & 4.35 & 0.02 \\
\hline Difference (post-pre) & \multicolumn{2}{|c|}{$9.11 \pm 2.97^{*}$} & \multicolumn{2}{|c|}{$8.37 \pm 1.76^{+}$} & \multicolumn{2}{|c|}{$6.20 \pm 1.75$} & & \\
\hline $\mathrm{t}$ & \multicolumn{2}{|c|}{-9.18} & \multicolumn{2}{|c|}{-13.40} & \multicolumn{2}{|c|}{-11.19} & & \\
\hline $\mathrm{p}$ & \multicolumn{2}{|c|}{0.00} & \multicolumn{2}{|c|}{0.00} & \multicolumn{2}{|c|}{0.00} & & \\
\hline TUG (second) & $35.11 \pm 6.47$ & $21.67 \pm 7.39$ & $36.50 \pm 17.27$ & $23.88 \pm 15.86$ & $36.70 \pm 4.71$ & $30.70 \pm 5.29$ & 6.07 & 0.01 \\
\hline Difference (post-pre) & \multicolumn{2}{|c|}{$-13.44 \pm 5.00^{*}$} & \multicolumn{2}{|c|}{$-12.62 \pm 6.45^{\dagger}$} & \multicolumn{2}{|c|}{$-6.00 \pm 3.85$} & & \\
\hline $\mathrm{t}$ & \multicolumn{2}{|c|}{8.06} & \multicolumn{2}{|c|}{5.53} & \multicolumn{2}{|c|}{4.92} & & \\
\hline $\mathrm{p}$ & \multicolumn{2}{|c|}{0.00} & \multicolumn{2}{|c|}{0.00} & \multicolumn{2}{|c|}{0.00} & & \\
\hline 10MWT (second) & $30.56 \pm 9.32$ & $17.44 \pm 7.10$ & $30.88 \pm 17.20$ & $18.75 \pm 11.83$ & $31.20 \pm 5.75$ & $25.40 \pm 4.90$ & 3.46 & 0.04 \\
\hline Difference (post-pre) & \multicolumn{2}{|c|}{$-13.10 \pm 6.75^{*}$} & \multicolumn{2}{|c|}{$-12.10 \pm 9.65^{\dagger}$} & \multicolumn{2}{|c|}{$-5.80 \pm 1.47$} & & \\
\hline $\mathrm{t}$ & \multicolumn{2}{|c|}{5.82} & \multicolumn{2}{|c|}{3.55} & \multicolumn{2}{|c|}{12.40} & & \\
\hline $\mathrm{p}$ & \multicolumn{2}{|c|}{0.00} & \multicolumn{2}{|c|}{0.01} & \multicolumn{2}{|c|}{0.00} & & \\
\hline
\end{tabular}

* significant difference $(\mathrm{p}<0.05)$ between aquatic treadmill and control; " significant difference $(\mathrm{p}<0.05)$ between anti-gravity treadmill and control. No significant difference was found in the comparison between aquatic treadmill group and anti-gravity treadmill group.

BBS: Berg balance scale, TUG: timed up and go, 10MWT: 10meter walking test.

and control were all homogenous (Table 1).

\section{Changes in balance and gait abilities of participants in this study}

The BBS, TUG, and 10MWT significantly increased in the aquatic treadmill group and anti-gravity treadmill group $(\mathrm{p}<0.05)$, and control group were significantly increased post intervention $(\mathrm{p}<0.05)$. In addition, aquatic treadmill group and anti-gravity treadmill group had significantly improved in BBS, TUG, and 10MWT scores compared with control group $(\mathrm{p}<0.05)$. But, no significant difference was found in the comparison between the aquatic treadmill and the antigravity treadmill group (Table 2).

\section{DISCUSSION}

Stroke patients have lower limit of stability due to greater body sway of standing position than normal subjects. This posture control consumes 1.5-2 times more energy than normal. ${ }^{19}$ As a results, stroke patients have increased risk of falls based on their asymmetric walking ability and balance ability. ${ }^{20}$ Hesse et al. ${ }^{21}$ noted that treadmill training using partial weight support could lead to improved walking speed based on symmetrical weight shifts. In this study, we investigated the change of balance ability and walking ability according to gait training by using underwater treadmill and anti-gravity treadmill as partial weight support type. Water reduces 
the stress on the joints through buoyancy, which counteracts the gravity felt by the human body on the land, which makes it possible to move with less force compared with the ground motion. Also, water with a higher viscosity than air has sufficient resistance during movement, which makes it easier to improve muscle strength. ${ }^{22,23}$ This evidence suggests that underwater walking training is possible with a small force compared with the ground walking training as shown by the results of Masumoto et al. ${ }^{24}$ In the study by Barela et al. ${ }^{25}$ changes in the weight support ratio due to buoyancy showed a change of ground reaction force, which was supported by the increase in muscle activity due to water viscosity. Tasuku et al. ${ }^{26}$ also suggested the basis of effective exercise by analyzing the relationship between viscosity and underwater walking speed. Based on this evidence, Park et al. ${ }^{12}$ compared gait training with aquatic treadmill and general treadmill. The results of this study showed symmetrical gait pattern based on improvement of stability in aquatic treadmill compared with general treadmill. The results of Jung et al. ${ }^{15}$ also showed that the increase in the stability of the stance phase resulted in an effective weight shift. These changes are thought to have not only improved balance ability but also psychological stability due to stability improvement led to improvement of walking ability. ${ }^{27}$ This evidence can be seen in the results of this study. The results of this study showed that the balance and gait ability were significantly improved compared with the control group. These changes may have been possible by helping the various characteristics of water and psychological stability to move more naturally.

In clinical practice, improvement of walking ability for stroke patients is an important index of rehabilitation research. ${ }^{28}$ Among them, walking speed can measure the level of functional recovery that can perform everyday life. ${ }^{29}$ It is possible to refer to walking training using a treadmill as an effective method of improving the walking ability. The gait training using a treadmill extends the weight support time of stance phase of affected side and leads to a symmetrical gait pattern. ${ }^{30}$ This symmetrical form of gait leads to a significant increase in walking speed. ${ }^{31}$ In addition, such treadmill training has been studied variously for the advantages of the control of the walking speed and the safe gait training. ${ }^{28}$ Nam et al. ${ }^{32}$ studied the balance and gait according to the presence and position of the treadmill handle, which is a topic related to the psychological stability of the subject. As a result of the study, it was found that the balance and walking ability were effective on the presence or absence of handles which can give psychological stability. In this study it was used conducted anti-gravity treadmill that has the advantages of safe gait training and can control weight support through objective indicators. Lathan et al. ${ }^{16}$ demonstrated that the use of an anti-gravity treadmill is an effective intervention method for restoring the ability of walking in stroke patients. Studies have shown that subjects using anti-gravity treadmill have been able to make effective changes in a variety of gait factors, including walking speed and stride length. The results of this study are similar to those of the previous study. The experimental group using anti-gravity treadmill showed a significant difference in balance ability and walking ability compared with the control group. However, no significant difference was found in the comparison between the aquatic treadmill and the anti-gravity treadmill group. This suggests that psychological stability of gait training has the same effect on the results in both groups. And the short period of 4 weeks for improving the muscle strength using the viscosity among the characteristics of the water was considered to be insufficient in order to statistically evaluate the difference between the two groups. Also, the results of the study could not be generalized because a large number of subjects were not included. In the future study, I would like to apply the training period for a long time and study through various subjects.

\section{REFERENCES}

1. Yang DJ, Park SK, Kang JI et al. Effects of changes in postural alignment on foot pressure and balance of patients with stroke. J Kor Phys Ther. 2014;26(4):226-33.

2. Horak FB. Postural orientation and equilibrium: what do we need to know about neural control of balance to prevent falls? Age Ageing. 2006; 35(Suppl 2):7-11.

3. Belda-Lois JM, Mena-del Horno S, Bermejo-Bosch I et al. Rehabilitation of gait after stroke: a review towards a top-down approach. J Neuroeng Rehabil. 2011;8:66.

4. Chang J, Lee S, Lee M et al. The correlations between gait speed and muscle activation or foot pressure in stroke patients. J Kor Soc Phys Ther. 2009;21(3):47-52.

5. Lee MS, Lee JH, Park SG et al. The effect of ankle joint taping applied to patients with hemiplegia on their gait velocity and joint angles. J Korean Soc Phys Ther. 2012;24(2):157-62.

6. Tyson SF, Hanley M, Chillala J et al. Balance disability after stroke. Phys Ther. 2006;86(1):30-8.

7. Kim CM, Eng JJ. Symmetry in vertical ground reaction force is accompanied by symmetry in temporal but not distance variables of gait in persons with stroke. Gait Posture. 2003;18(1):23-8.

8. Park RJ, Kim SS, Kim YG et al. The effects of aqua-exercise on the bal- 
ance of one leg stance in the elderly women. J Korean Soc Phys Ther. 2002;14(1):89-98.

9. Vivas J, Arias P, Cudeiro J. Aquatic therapy versus conventional landbased therapy for Parkinson's disease: an open-label pilot study. Arch Phys Med Rehabil. 2011; 92(8):1202-10.

10. Melzer I, Elbar O, Tsedek I et al. A water-based training program that include perturbation exercises to improve stepping responses in older adults: study protocol for a randomize. BMC Geriatrics. 2008;8:19.

11. Masumoto K, Shono T, Hotta N et al. Muscle activation, cardiorespiratory response, and rating of perceived exertion in older subjects while walking in water and on dry land. J Electromyogr Kinesiol. 2008;18(4):581-90.

12. Park J, Lee D, Lee S et al. Comparison of the effects of exercise by chronic stroke patients in aquatic and land environments. J Kor Phys Ther. 2011; 23(5):821-4.

13. Arnold CM, Busch AJ, Schachter CL et al. A randomized clinical trial of aquatic versus land exercise to improve balance, function, and quality of life in older women with osteoporosis. Physiother Can. 2008;60(4):296306.

14. Hinman RS, Heywood SE, Day AR. Aquatic physical therapy for hip and knee osteoarthritis: results of a single-blind randomized controlled trial. Phys Ther. 2007;87(1):32-43.

15. Jung T, Lee D, Charalambous $C$ et al. The influence of applying additional weight to the affected leg on gait patterns during aquatic treadmill walking in people poststroke. Arch Phys Med Rehabil. 2010;91(1):12936.

16. Lathan C, Myler A, Bagwell J et al. Pressure-controlled treadmill training in chronic stroke: a case study with AlterG. J Neurol Phys Ther. 2015; 39(2):127-33.

17. Berthelsen MP, Husu E, Christensen SB et al. Anti-gravity training improves walking capacity and postural balance in patients with muscular dystrophy. Neuromuscul Disord. 2014;24(6):492-8.

18. Mercer JA, Applequist B, Masumoto K et al. Muscle activity during running with different body weight support mechanisms: aquatic environment versus body weight support treadmill. J Sport Rehabil. 2014;23(4): 300-6.

19. Geiger RA, Allen JB, O'Keefe J et al. Balance and mobility following stroke: effects of physical therapy interventions with and without bio- feedback/forceplate training. Phys Ther. 2001;81(4):995-1005

20. Srivastava A, Taly AB, Gupta A et al. Post-stroke balance training: role of force platform with visual feedback technique. J Neurol Sci. 2009;287(12):89-93.

21. Hesse S. Treadmill training with partial body weight support after stroke: a review. Neuro Rehabilitation. 2008;23(1):55-65.

22. Becker BE. Aquatic therapy: scientific foundations and clinical rehabilitation applications. PM R. 2009;1(9):859-72.

23. Chevutschi A, Alberty M, Lensel G et al. Comparison of maximal and spontaneous speeds during walking on dry land and water. Gait Posture. 2009;29:403-7.

24. Masumoto K, Takasugi S, Hotta $\mathrm{N}$ et al. Electromyographic analysis of walking in water in healthy humans. J Physiol Anthropol Appl Human Sci. 2004;23(4):119-27.

25. Barela AM, Stolf SF, Duarte M. Biomechanical characteristics of adults walking in shallow water and on land. J Electromyogr Kinesiol. 2006; 16(3):250-6.

26. Miyoshi T, Shirota T, Yamamoto S. Functional roles of lower-limb joint moments while walking in water. Clin Biomech. 2005;20(2):194-201.

27. Kim SH, Lee DK, Kim EK. Effect of aquatic exercise on balance and depression of stroke patients. J Kor Phys Ther. 2014:26(2):104-9.

28. Ada L, Dean CM, Hall JM et al. A treadmill and overground walking program improves walking in persons residing in the community after stroke: a placebo-controlled, randomized trial. Arch Phys Med Rehabil. 2003;84(10):1486-91.

29. Kim CM, Eng JJ. The relationship of lower-extremity muscle torque to locomotor performance in people with stroke. Phys Ther. 2003;83(1):4957.

30. Chen G, Patten C. Treadmill training with harness support: selection of parameters for individuals with poststroke hemiparesis. J Rehabil Res Dev. 2006;43(4):485-98.

31. Patterson SL, Rodgers MM, Macko RF et al. Effect of treadmill exercise training on spatial and temporal gait parameters in subjects with chronic stroke: a preliminary report. J Rehabil Res Dev. 2008;45(2):221-8.

32. Nam SH, Kang KW, Kwon JW et al. The effects of handrails during treadmill gait training in stroke patients. J Korean Soc Phys Ther. 2013; 25(1):25-8. 